\title{
SOLUTION OF THE PROBLEM OF SCATTERING OF WATER WAVES BY A NEARLY VERTICAL PLATE
}

\author{
L. VIJAYA BHARATHI ${ }^{1}$, A. CHAKRABARTI ${ }^{1}$, B. N. MANDAL ${ }^{2}$ and \\ S. BANERJEE ${ }^{3}$
}

(Received 21 February 1991; revised 14 February 1992)

\begin{abstract}
An approximate solution is determined for the problem of scattering of water waves by a nearly vertical plate, by reducing it to two mixed boundary-value problems (BVP) for Laplace's equation, using perturbation techniques. While the solution of one of these BVP is well-known, the other BVPs is reduced to the problem of solving two uncoupled Riemann-Hilbert problems, and the complete solution of the problem under consideration up to first-order accuracy is derived with a special assumption on the shape of the plate and a related approximation. Known results involving the reflection and transmission coefficients are reproduced.
\end{abstract}

\section{Introduction}

The problem of scattering of water waves by a partially immersed nearly vertical barrier was first considered by Shaw [7]. Various methods of solution have been presented later for the approximate solution of this class of water wave problems $[2,10,3]$. In the present paper we have considered the scattering problem involving a nearly vertical submerged plate of finite length in deep water. This problem has already been handled for solution by Mandal and Kundu [3] by using the Green's function-cum-integral integral approach. A relatively shorter route involving the direct utility of Green's identities, as employed by Mandal and Chakrabarti [2], has also been used by Mandal and Kundu [3] to derive results

\footnotetext{
${ }^{1}$ Department of Mathematics, Indian Institute of Science, Bangalore-12, India.

${ }^{2}$ Physical and Earth Sciences Division, Indian Statistical Institute, Calcutta-35, India.

${ }^{3}$ Calcutta Mathematical Society, Calcutta-9, India.

(C) Australian Mathematical Society, 1994, Serial-fee code 0334-2700/94
} 
involving first-order corrections to the reflection and transmission coefficients. Though the final important results derived earlier as well as in the present paper are the first-order corrections to the reflection and transmission coefficients associated with the nearly vertical plate under consideration, explicit results for the velocity potential of the flow problem, even up to the first-order terms, have not been derived so far. Determination of the full potential (up to first order) is, therefore, one of the many motives behind taking up the present study, to which a direct complex variable method is observed to be well suited. The complex variable method utilised here is similar to the one used to handle the problem of a submerged nearly vertical barrier, by Vijaya Bharathi and Chakrabarti [10]. This paper will be referred to as Paper 1 in the text that follows.

Using a perturbation approach, the problem of determining the velocity potential $\phi(x, y)$ associated with the nearly vertical plate is reduced, up to the first-order approximation, to that of determining two potential functions $\phi_{0}(x, y)$ and $\phi_{1}(x, y)$ under certain boundary conditions (BC), prescribed along the horizontal boundary, $y=0$ and $-\infty<x<\infty$ and along the vertical line $x=0, y \in[-b,-a]$. It is observed that the $\mathrm{BC}$ to be satisfied by the function $\phi_{0}(x, y)$ are the same as the ones encountered in the case of a plane vertical plate problem whose solution is available in the literature (see $[4,1,9]$ and others), whereas the $\mathrm{BC}$ for the function $\phi_{1}$ are of a new type. It is important to realise that $\phi_{0}$ is a transmission potential for a fixed vertical plate of finite length, whereas $\phi_{1}$ is a radiation potential for a related plane vertical wave-maker problem.

This new BVP will be solved in Section 2 of the present paper by employing a complex variable technique as described in Paper 1. Introducing a reduced complex potential $W(z)$ (see [1] and [6]), the problem in question is shown to be equivalent to a pair of uncoupled Riemann-Hilbert problems for the determination of two sectionally analytical functions $\lambda(z)$ and $\mu(z)$, analytic in the entire complex $z(=x+j y)$ plane cut along the segments $[-j b,-j a]$ and $[j a, j b]$. The complex potential $W(z) \equiv \phi_{1}(x, y)+j \psi_{1}(x, y)\left(\psi_{1}\right.$ is the conjugate of $\left.\phi_{1}\right)$, is then obtained by straightforward integration. The asymptotic relations on the complex potential $W(z)$ are then used to derive the two important constants $R_{1}$ and $T_{1}$, (see Paper 1 also) representing the first-order corrections to the reflection and transmission coefficients, and these are found to be the same as the ones obtained by Mandal and Kundu [3] recently. The formal explicit expressions for the near-field potential $\phi_{1}(x, y)$ are also determined by using a standard contour-integration procedure. 


\section{Formulation of the problem}

With the usual assumptions of linear water wave theory (see Stoker [8]), the main problem under consideration is that of the determination of the velocity potential $\phi(x, y)$ that corresponds to the irrotational motion of water in the region $y \leq 0$, associated with the scattering of simple harmonic progressive waves which are incident normally on the nearly vertical plate, the shape of which is described by the equation $x=\varepsilon C(y)$ for $y \in[-b,-a]$ (where $\varepsilon \ll 1$ and $\max C(y)=1$ ) and $C(-a)=0$.

The governing equations and conditions to be satisfied by $\phi(x, y)$ are as follows (see [7, 2]):

$$
\begin{aligned}
& \text { (i) } \frac{\partial^{2} \phi}{\partial x^{2}}+\frac{\partial^{2} \phi}{\partial y^{2}}=0, \quad(y<0,-\infty<x<\infty) \\
& \text { (ii) } K \phi-\frac{\partial \phi}{\partial y}=0, \quad \text { on } y=0 \\
& \text { (iii) } \frac{\partial \phi}{\partial x} \varepsilon \frac{\partial}{\partial y}\left[C(y) \frac{\partial \phi}{\partial y}\right], \quad \text { on } x=0^{ \pm}, y \in[-b,-a] \\
& \text { (iv) } r^{1 / 2}|\nabla \phi| \rightarrow 0, \quad \text { as } r^{2}=\left(x^{2}+(y+d)^{2}\right) \rightarrow 0(d=a \text { or } b) \\
& \text { (v) } \phi(x, y) \sim \begin{cases}T e^{i K x+K y} & \text { as } x \rightarrow \infty \\
e^{i K x+K y}+R e^{-i K x+K y} & \text { as } x \rightarrow-\infty .\end{cases}
\end{aligned}
$$

and

$$
\text { (vi) } \phi, \quad \frac{\partial \phi}{\partial y} \rightarrow 0, \text { as } y \rightarrow-\infty,
$$

where $K=\sigma^{2} / g, g$ is the acceleration due to gravity and the harmonic time dependence $e^{-i \sigma t}$ ( $\sigma$ represents the circular frequency) is dropped throughout the paper. The complex constants $R$ and $T$ are the reflection and transmission coefficients of the plate, which are to be determined.

The BVP is attacked for approximate solution by using perturbational series (see [2] and [3]), as described next. We assume

$$
\begin{aligned}
\phi(x, y) & =\phi_{0}(x, y)+\varepsilon \phi_{1}(x, y)+O\left(\varepsilon^{2}\right), \\
R & =R_{0}+\varepsilon R_{1}+O\left(\varepsilon^{2}\right) \\
\text { and } & \\
T & =T_{0}+\varepsilon T_{1}+O\left(\varepsilon^{2}\right) .
\end{aligned}
$$


The problem of solving $\phi(x, y)$ satisfying the conditions as given by the relations (i) and (vi) then reduces, up to $\mathrm{O}(\varepsilon)$, to that of determining the two potentials $\phi_{0}(x, y)$ and $\phi_{1}(x, y)$ which will be referred to as BVP 1 and BVP 2 respectively.

BVP 1. The $\mathrm{BC}$ to be satisfied by the potential $\phi_{0}(x, y)$ are the same as those involving the plane vertical plate problem (see [1]), the solution of which is as given as below. (See [3])

$$
\begin{array}{rlrl}
\phi_{0}(x, y)= & T_{0} e^{K y+i K x}-D \int_{0}^{\infty} \frac{J(\eta) e^{-\eta x}}{\eta^{2}+K^{2}}[\eta \cos \eta y+K \sin \eta y] d \eta, & & \text { for } x>0, \\
=e^{K y+i K x}+ & R_{0} e^{K y-i K x} \\
& +D \int_{0}^{\infty} \frac{J(\eta) e^{+\eta x}}{\eta^{2}+K^{2}}[\eta \cos \eta y+K \sin \eta y] d \eta, & & \text { for } x<0,
\end{array}
$$

with

$$
D=\frac{i}{\alpha-\beta-i \gamma}, \quad T_{0}=\frac{\alpha-\beta}{\alpha-\beta-i \gamma} \quad \text { and } \quad R_{0}=\frac{-i r}{\alpha-\beta-i \gamma},
$$

where

$$
\begin{aligned}
& \alpha=d^{2} a_{2}(K)-a_{2}^{\prime \prime}(K), \quad \beta=d^{2} a_{3}(K)-a_{3}^{\prime \prime}(K), \\
& \gamma=d^{2} a_{1}(K)-a_{1}^{\prime \prime}(K), \quad d^{2}=a_{1}^{\prime \prime}(-K) / a_{1}(K)
\end{aligned}
$$

and

$$
J(\eta)=\frac{2}{\pi} \int_{a}^{b} \frac{d^{2}-t^{2}}{\rho(t)} \sin (t \eta) d t
$$

where

$$
\begin{aligned}
a_{1}(K, F) & =\int_{a}^{b} \frac{e^{-K t}}{\rho(t)} F(t) d t, & a_{2}(K, F) & =\int_{-a}^{a} \frac{e^{-K t} F(t)}{\left[\left(a^{2}-t^{2}\right)\left(b^{2}-t^{2}\right)\right]^{1 / 2}} d t, \\
a_{1}(-K, F) & =\int_{-a}^{-b} \frac{e^{-K t}}{\rho(t)} F(t) d t, & a_{3}(K, F) & =\int_{b}^{\infty} \frac{e^{-K t} F(t)}{\left[\left(t^{2}-a^{2}\right)\left(t^{2}-b^{2}\right)\right]^{1 / 2}} d t
\end{aligned}
$$

and

$$
\begin{aligned}
& a_{m}(K)=a_{m}(K, 1) ; \quad a_{m}^{\prime \prime}(K)=\frac{d^{2}}{d K^{2}} a_{m}(K) \quad \text { where } m=1,2 \text { or } 3 \\
& a_{1}(-K)=a_{1}(-K, 1) ; \quad a_{1}^{\prime \prime}(-K)=\frac{d^{2}}{d K^{2}} a_{1}(-K)
\end{aligned}
$$


and

$$
\rho(t)=\left[\left(b^{2}-t^{2}\right)\left(t^{2}-a^{2}\right)\right]^{1 / 2}
$$

with $F(t)$ being any function such that the above integrals are well defined.

BVP 2. The BVP for the function $\phi_{1}(x, y)$ is governed by the following equations and conditions:

$$
\begin{aligned}
& \text { (i) } \frac{\partial^{2} \phi_{1}}{\partial x^{2}}+\frac{\partial^{2} \phi_{1}}{\partial y^{2}}=0, \quad(y<0,-\infty<x<\infty) \\
& \text { (ii) } K \phi_{1}-\frac{\partial \phi_{1}}{\partial y}=0, \quad \text { on } y=0 \\
& \text { (iii) }\left.\frac{\partial \phi_{1}}{\partial x}\right|_{x=0^{ \pm}}=\frac{\partial}{\partial y}\left[C(y) \frac{\partial \phi_{0}}{\partial y}\left(0^{ \pm}, y\right)\right], \quad \text { for } y \in[-b,-a] \\
& \text { (iv) } \quad r^{1 / 2}\left|\nabla \phi_{1}\right| \rightarrow 0, \quad \text { as } r \rightarrow 0 \\
& \text { (v) } \quad \phi(x, y) \sim \begin{cases}R_{1} e^{K y-i K x} & \text { as } x \rightarrow-\infty \\
T_{1} e^{K y+i K x} & \text { as } x \rightarrow+\infty\end{cases}
\end{aligned}
$$

and

$$
\text { (vi) } \phi_{1}, \frac{\partial \phi_{1}}{\partial y} \rightarrow 0, \quad \text { as } y \rightarrow-\infty .
$$

We shall describe, in the next section, the complex variable technique employed to solve this problem.

\section{The method of solution of BVP 2}

We introduce the reduced potential (see Paper 1), as defined by

$$
W(z)=\frac{d w}{d z}+j K w,
$$

where

$$
w(z)=\phi_{1}(x, y)+j \psi_{1}(x, y)
$$

is the complex potential and $j$ is a second imaginary unit not interacting with the unit $i$, occurring in the earlier relations. The function $\psi_{1}(x, y)$ is a function which is the harmonic conjugate of $\phi_{1}(x, y)$.

Then, using the Cauchy-Riemann equations in the $\mathrm{BC}$ (ii) and (iii) on the function $\phi_{1}(x, y)$, we obtain the following conditions for the function $W(z)$ :

$$
\operatorname{Im}_{j} W(z)=0 \quad \text { on } y=0
$$


and

$$
\lim _{x \rightarrow 0^{ \pm}} \operatorname{Re} W(z)=\frac{\partial \phi_{1}}{\partial x}-K \psi_{1}=f_{ \pm}(y) \quad \text { for } y \text { on } L,
$$

where

$$
f_{ \pm}(y)=\left(\frac{\partial}{\partial y}-K\right)\left[C(y) \frac{\partial \phi_{0}}{\partial y}\left(0^{ \pm}, y\right)\right] \quad \text { for } y \text { on } L
$$

$L$ representing the line segment $x=0,-b<y<-a$.

The sectionally analytic function $W(z)$ (analytic in the $z$-plane, cut along the segment $L$ and bounded by the line $y=0$ ) in the half-plane $y<0$ must meet with the infinity requirement that

$$
|W(z)|<\infty
$$

and the edge conditions that

$$
|W(z)|=O\left(r^{-1 / 2}\right) \quad \text { as } r \rightarrow 0,
$$

with $r^{2}=x^{2}+(y+d)^{2},(d=a$ or $b)$.

The above conditions on $W(z)$ follow directly from the requirements (iv), (v) and (vi) to be satisfied by the function $\phi_{1}(x, y)$.

We now define a function $W_{1}(z)$ which is sectionally analytic in the whole of the $z$-plane cut along $L \cup L^{\prime}\left(L^{\prime}\right.$ is the reflection of $L$ into the plane $y>0$, with respect to the $x$-axis) by using Schwarz's reflection principle in the following manner,

$$
W_{1}(z)= \begin{cases}W(z) & \text { for } y \leq 0 \\ \overline{W(\bar{z})} & \text { for } y>0\end{cases}
$$

From the above definition (3.8) of $W_{1}(z)$, we observe that,

$$
W_{1}(-z)=\overline{W_{1}(-\bar{z})}
$$

The conditions on $W(z)$ as given by (3.3), (3.4), (3.6) and (3.7) give rise to the following requirements to be satisfied by $W_{1}(z)$ :
(I)
$\operatorname{Im}_{j} W_{1}(z)=0$
on $y=0$
(II) $\quad \operatorname{Re}_{j} \lim _{x \rightarrow 0^{ \pm}} W_{1}(z)=f_{ \pm}(-|y|)$ for $y$ on $L \cup L^{\prime}$
(III) $\left|W_{1}(z)\right|<\infty$

in the entire cut $z$-plane and

$$
\left|W_{1}(z)\right| \sim O\left(r^{-1 / 2}\right) \text { as } r \rightarrow 0
$$


where $r^{2}=x^{2}+(y+d)^{2}, d= \pm a$ or $\pm b$.

Next, in order to solve the problem for the function $W_{1}(z)$ satisfying the conditions (I) to (IV), we define the two sectionally analytic functions $\lambda(z)$ and $\mu(z)$ as given by (see Paper 1):

$$
\lambda(z)=\frac{1}{2}\left[W_{1}(z)+\overline{W_{1}(-\bar{z})}\right] \text { and } \mu(z)=\frac{1}{2}\left[W_{1}(z)-\overline{W_{1}(-\bar{z})}\right] .
$$

On using the following standard notations for the limiting values,

$$
\lim _{x \rightarrow 0^{ \pm}} \lambda(z)= \begin{cases}\lambda^{ \pm}(j y) & \text { for } y \text { on } L \\ \lambda^{\mp}(j y) & \text { for } y \text { on } L^{\prime}\end{cases}
$$

the $\mathrm{BC}(\mathrm{II})$ on $W_{1}(z)$ can be expressed as

$$
\lambda^{+}(j y)+\lambda^{-}(j y)=g(-|y|) \quad \text { for } y \text { on } L \cup L^{\prime}
$$

and

$$
\mu^{+}(j y)-\mu^{-}(j y)= \begin{cases}f(y) & \text { for } y \text { on } L \\ -f(-y) & \text { for } y \text { on } L^{\prime}\end{cases}
$$

where

$$
\left.\begin{array}{l}
g(y)=f_{+}(y)+f_{-}(y) \\
f(y)=f_{+}(y)-f_{-}(y)
\end{array}\right\} \text { for } y \in(-b,-a) .
$$

It follows from (3.10) that the sectionally analytic functions in the cut $z$-plane also satisfy conditions similar to the ones as given by the equations (III) and (IV) on $W_{1}(z)$.

We thus have two independent Riemann-Hilbert problems for the functions $\lambda(z)$ and $\mu(z)$ with the $\mathrm{BC}(3.11)$ to be satisfied on $L \cup L^{\prime}$. The solution of these problems can be obtained by using the techniques available in Muskhlelishvili [5], in the following form:

$$
\lambda(z)=\lambda_{0}(z)\left[P(z)-\frac{1}{\pi} \int_{-a}^{-b} \frac{\eta}{\eta^{2}+z^{2}} g(\eta) \rho(\eta) d \eta\right]
$$

and

$$
\mu(z)=Q(z)-\frac{z}{\pi} \int_{-a}^{-b} \frac{f(\eta)}{\eta^{2}+z^{2}} d \eta
$$

where $P(z)$ and $Q(z)$ are polynomials in $z$ and $\rho(\eta)$ is as defined by (2.4) and

$$
\lambda_{0}(z)=\left[\left(z^{2}+a^{2}\right)\left(z^{2}+b^{2}\right)\right]^{-1 / 2} .
$$


Using (3.10) and the infinity conditions (III) on $W_{1}(z)$, we obtain the following forms of the polynomials $P(z)$ and $Q(z)$ :

$$
P(z)=C_{0}+C_{1} z+C_{2} z^{2} \quad Q(z)=Q_{0} .
$$

Then using (3.9) on $W_{1}(z)$ and (3.10), after simplification we arrive at the following identity:

$$
Q_{0}=C_{1} \lambda_{0}(z) \quad \text { for all } z,
$$

and we thus decide that

$$
Q_{0}=C_{1}=0 .
$$

A similar procedure ensures that the constants $C_{0}$ and $C_{2}$ will have to be real constants, $A$ and $B$ respectively, in order to satisfy the condition (I) on $W_{1}(z)$.

We have therefore ultimately determined the function $W_{1}(z)$. completely except for two arbitrary real constants $A$ and $B$, and we have that

$W_{1}(z)=\lambda_{0}(z)\left[A+B z^{2}-\frac{1}{\pi} \int_{-a}^{-b} \frac{\eta}{\eta^{2}+z^{2}} g(\eta) \rho(\eta) d \eta\right]-\frac{z}{\pi} \int_{-a}^{-b} \frac{f(\eta)}{\eta^{2}+z^{2}} d \eta$.

Upon integrating (3.1) and using (3.17), we then obtain the complex potential $W(z)$ as given by :

$$
W(z)=e^{-j K z}\left[C+\int_{-a j}^{z} e^{j k \xi} W_{1}(\xi) d \xi\right],
$$

where $C$ is an arbitrary real constant. This choice of $C$ satisfies the condition on $\operatorname{Im}_{j} W(z)$ i.e. $\psi_{1}(x, y)$ on $L$ which is derived from the condition (iii) on $\phi_{1}(x, y)$.

\section{Determination of the constants $A, B, C, R_{1}$ and $T_{1}$}

It follows from (3.2) and (3.18), along with (3.17) for $W_{1}(z)$ that the potential $\phi_{1}(x, y)\left(=\operatorname{Re}_{j} W(z)\right)$ is determined completely except for the arbitrary constants $A, B$ and $C$ which are real with respect to $j$. Also the constants $R_{1}$ and $T_{1}$, the first-order corrections to the reflection and transmission coefficients (Shaw [7]) are to be determined explicitly. For this purpose we carry out the following asymptotic analysis.

We first observe that

$$
\left|W_{1}(z)-B\right| \rightarrow 0, \quad \text { as }|z| \rightarrow \infty
$$


Using (4.1) along with Cauchy's integral theorem, (see Paper 1 and [1]) we can derive the following relation, as $x \rightarrow+\infty$ :

$$
\begin{array}{r}
\int_{-a j}^{z} e^{j k \xi} W_{1}(\xi) d \xi \sim \frac{-j B}{K} e^{j K z}+\Delta+j \lambda_{1}+A a_{1}(k)-B a_{1}^{\prime \prime}(k)-a_{1}(K, F) \\
+j\left[A\left(a_{2}(k)-a_{3}(k)\right)-B\left(a_{2}^{\prime \prime}(k)-a_{3}^{\prime \prime}(k)\right)+a_{3}(K, F)-a_{2}(K, F)\right]
\end{array}
$$

and, as $x \rightarrow-\infty$, we find that

$$
\begin{aligned}
\int_{-a j}^{2} e^{j k \xi} W_{1}(\xi) d \xi \sim \frac{-j B}{K} e^{j K z}+\Delta+j \lambda_{2}-A a_{1}(k)+B a_{1}^{\prime \prime}(k)+a_{1}(K, F) \\
+j\left[A\left(a_{2}(k)-a_{3}(k)\right)-B\left(a_{2}^{\prime \prime}(k)-a_{3}^{\prime \prime}(k)\right)-a_{2}(K, F)+a_{3}(K, F)\right],
\end{aligned}
$$

where

$$
\left.\begin{array}{rl}
\Delta & =\frac{1}{\pi} \int_{-a}^{\infty} t e^{-K t} d t \int_{-a}^{-b} \frac{f(\eta)}{\eta^{2}-t^{2}} d \eta \\
(t) & =\frac{1}{\pi} \int_{-a}^{-b} \frac{\eta g(\eta) \rho(\eta)}{\eta^{2}-t^{2}} d \eta \\
\lambda_{1} & =\int_{a}^{b} e^{-K t} f_{+}(-t) d t \\
\lambda_{2} & =\int_{a}^{b} e^{-K t} f_{-}(-t) d t,
\end{array}\right\}
$$

and the other constants $a_{m}(K), a_{m}^{\prime \prime}(K)$ and $a_{m}(K, F)$ are as defined earlier by (2.3), for $m=1,2,3$.

We can, therefore, obtain from the asymptotic relations (4.2) and (4.3) the behaviour of the complex potential $W(z)$ and derive the following behaviour of the function $\phi_{1}(x, y)$ [using (3.2) that $\operatorname{Re}_{j} W(z)=\phi_{1}(x, y)$ ] as $x \rightarrow \pm \infty$ :

$$
\begin{array}{r}
\phi_{1}(x, y) \sim \quad e^{K y} \cos K x\left[C+\Delta+A a_{1}(K)-B a_{1}^{\prime \prime}(K)-a_{1}(K, F)\right] \\
+e^{K y} \sin K x\left[\lambda_{1}+A\left(a_{2}(K)-a_{3}(K)\right)\right. \\
\left.-B\left(a_{2}^{\prime \prime}(K)-a_{3}^{\prime \prime}(K)\right)-a_{2}(K, F)+a_{3}(K, F)\right], \\
\text { as } x \rightarrow+\infty
\end{array}
$$

and

$$
\begin{array}{r}
\phi_{1}(x, y) \sim \quad e^{K y} \cos K x\left[C+\Delta-A a_{1}(K)+B a_{1}^{\prime \prime}(K)+a_{1}(K, F)\right] \\
+e^{K y} \sin K x\left[\lambda_{2}+A\left(a_{2}(K)-a_{3}(K)\right)\right. \\
\left.-B\left(a_{2}^{\prime \prime}(K)-a_{3}^{\prime \prime}(K)\right)-a_{2}(K, F)+a_{3}(K, F)\right] \\
\text { as } x \rightarrow-\infty
\end{array}
$$


Comparing the above relations (4.6) and (4.7) with the conditions (V) on $\phi_{1}(x, y)$, we obtain that

$$
\begin{aligned}
T_{1} & =C+\Delta+A a_{1}(K)-B a_{1}^{\prime \prime}(K)-a_{1}(K, F), \\
i t_{1} & =\lambda_{1}+A\left[a_{2}(K)-a_{3}(K)\right]-B\left[a_{2}^{\prime \prime}(K)-a_{3}^{\prime \prime}(K)\right]-a_{2}(K, F)+a_{3}(K, F), \\
R_{1} & =C+\Delta-A a_{1}(K)+B a_{1}^{\prime \prime}(K)+a_{1}(K, F), \\
i R_{1} & =-\lambda_{2}-A\left[a_{2}(K)-a_{3}(K)\right]+B\left[a_{2}^{\prime \prime}(K)-a_{3}^{\prime \prime}(K)\right]+a_{2}(K, F)-a_{3}(K, F) .
\end{aligned}
$$

Now we must meet with the requirement that the velocity potential $\phi(x, y)$ and, therefore, the functions $\phi_{0}(x, y)$ and $\phi_{1}(x, y)$ are single-valued. Hence, circulation of the flow induced by the potential $\phi_{1}(x, y)$ around any closed contour must be zero. This criterion (see [1] and [6], also) gives rise to the relation that

$$
\operatorname{Re}_{j} \oint_{S} e^{j K \xi} W_{1}(\xi) d \xi=0
$$

where $S$ is a closed contour around $L$. Following Evans [1] and shrinking the contour onto $L$ we simplify the above relation to the following equation:

$$
A a_{1}(-K)-B a_{1}^{\prime \prime}(-K)-a_{1}(-K, F)=0,
$$

where $F(t)$ is as defined earlier by (4.4) and $a_{1}(-K), a_{1}^{\prime \prime}(-K)$ and $a_{1}(-K, F)$ are defined by (2.3).

The five relations, given by (4.8) and (4.9), provide us with five equations for the determination of the five unknowns $A, B, C, R_{1}$ and $T_{1}$ and we ultimately obtain the following expressions for these constants:

$$
\begin{aligned}
& A= B d^{2}+\left[a_{1}(-K, F) / a_{1}(-K)\right] \\
& B=(\alpha-\beta-i \gamma)^{-1}\left\{i a_{1}(K, F)-a_{2}(K, F)+a_{3}(K, F)+\frac{1}{2}\left(\lambda_{1}+\lambda_{2}\right)\right. \\
&\left.\quad \quad \quad\left[a_{1}(-K, F) / a_{1}(-K)\right]\left[i a_{1}(K)-a_{2}(K)+a_{3}(K)\right]\right\} \\
& C=\frac{i}{2}\left[\lambda_{2}-\lambda_{1}\right]-\Delta, \quad \\
& T_{1}=\frac{i}{2}\left[\lambda_{2}-\lambda_{1}\right]-a_{1}(K, F)+a_{1}(K)\left[a_{1}(-K, F) / a_{1}(-K)\right] \\
&+B\left[d^{2} a_{1}(K)-a_{1}^{\prime \prime}(K)\right] \\
& R_{1}=\frac{i}{2}\left[\lambda_{2}-\lambda_{1}\right]+a_{1}(K, F)-a_{1}(K)\left[a_{1}(-K, F) / a_{1}(-K)\right] \\
&+B\left[a_{1}^{\prime \prime}(K)-a_{1}(K) d^{2}\right]
\end{aligned}
$$

where the constants $\alpha, \beta, \gamma$ and $d^{2}$ are as defined earlier by (2.2). 
Using the above relations and the definitions of the constants $\lambda_{1}$ and $\lambda_{2}$ as given by (4.5), we derive that

$$
i\left(T_{1}+R_{1}\right)=2 K \int_{-a}^{-b} e^{K t} C(t) \frac{\partial}{\partial t} \tilde{\phi}_{0}(t) d t
$$

where

$$
\tilde{\phi}_{0}(y)=\phi_{0}\left(0^{+}, y\right)-\phi_{0}\left(0^{-}, y\right),
$$

after using a further assumption that $C(-b)=0$. But the relations $(2.1)$ and (2.2) for the function $\phi_{0}(x, y)$ give rise to the result that

$$
\tilde{\phi}_{0}(y)=\left\{\begin{array}{l}
\frac{2 i e^{K y}}{\alpha-\beta-i \gamma} \int_{-a}^{y} \frac{\left(d^{2}-t^{2}\right)}{\rho(t)} e^{-K t} d t, \quad-b<y<-a \\
0, \quad \text { otherwise. }
\end{array}\right.
$$

This relation (4.13) when substituted in (4.11) finally gives that

$$
\begin{gathered}
\frac{T_{1}+R_{1}}{4 K}[\alpha-\beta-i \gamma]=K \int_{-a}^{-b} e^{2 K t} C(t) d t \int_{-a}^{t} \frac{\left(d^{2}-\eta^{2}\right) e^{-k \eta}}{\rho(\eta)} d \eta \\
+\int_{-a}^{-b} \frac{\left(d^{2}-t^{2}\right)}{\rho(t)} C(t) e^{K t} d t
\end{gathered}
$$

If in the above result (4.14) we use the identity that $T_{1}=0$ which can be derived by employing physical arguments similar to the ones used by Shaw [7], we find that the expression for the constant $R_{1}$ agrees with that obtained by Mandal and Kundu [3].

\section{Determination of the near-field for $\phi_{1}(x, y)$}

Simplifying (3.18), we can obtain explicitly the complex potential $W(z)$ in the halfplane $y<0$. For $z=(x+j y)$ near the barrier, by choosing an appropriate contour (see Paper 1) and using the relations (4.2) and (4.8) we can arrive at the following expression for $\phi_{1}(x, y)$ for $x>0$ :

$$
\phi_{1}(x, y)=T_{1} e^{K y+i K x}-R e_{j} e^{-j K z} \int_{z}^{\infty} e^{j K \xi} W_{1}(\xi) d \xi .
$$

Substituting for $W_{1}(\xi)$ from (2.17), we obtain that

$$
\int_{z}^{\infty} e^{j K \xi} W_{1}(\xi) d \xi=S_{1}(z)-\frac{1}{\pi} S_{2}(z)-\frac{1}{\pi} S_{3}(z)
$$


where

$$
S_{1}(z)=B \int_{z}^{\infty} e^{j k \xi} \lambda_{0}(\xi)\left[l^{2}+\xi^{2}\right] d \xi
$$

with $l^{2}=A / B$,

$$
S_{2}(z)=\int_{z}^{\infty} \xi e^{j K \xi} d \xi \int_{-a}^{-b} \frac{f(\eta)}{\eta^{2}+\xi^{2}} d \eta
$$

and

$$
S_{3}(z)=\int_{z}^{\infty} e^{j K \xi} \lambda_{0}(\xi) d \xi \int_{-a}^{-b} \frac{\eta g(\eta)}{\eta^{2}+\xi^{2}} \rho(\eta) d \eta .
$$

We then simplify the above expressions, in the manner as described below.

From Laplace-transform theory we find that

$$
\int_{0}^{\infty} e^{-\xi \eta} M(\eta) d \eta=\left(l^{2}+\xi^{2}\right) \lambda_{0}(\xi)
$$

where

$$
M(\eta)=\frac{2}{\pi} \int_{a}^{b} \frac{\left(l^{2}-u^{2}\right)}{\rho(u)} \sin (u \eta) d u
$$

and $\lambda_{0}(\xi)$ is as defined in (3.14). Using the result (5.4), we can express the integral $S_{1}(z)$ as follows:

$$
\begin{aligned}
S_{1}(z) & =B \int_{z}^{\infty} e^{j K \xi} d \xi \int_{0}^{\infty} M(\eta) e^{-\xi \eta} d \eta \\
& =\int_{0}^{\infty} M(\eta) d \eta \int_{z}^{\infty} e^{(j K-\eta) \xi} \xi d \eta \\
& =B e^{j K z} \int_{0}^{\infty} \frac{M(\eta)}{\eta-j K} e^{-\eta z} d \eta
\end{aligned}
$$

Also, using the standard result that

$$
\int_{0}^{\infty} e^{-\xi \eta} \cos (t \eta) d \eta=\frac{\xi}{\xi^{2}+t^{2}}
$$

we can express $S_{2}(z)$ as

$$
S_{2}(z)=e^{j K z} \int_{-a}^{-b} f(t) d t \int_{0}^{\infty} \frac{\cos (t \eta)}{\eta-j K} e^{-\eta z} d \eta
$$


We next write the integral $S_{3}(z)$ in the following form:

$$
2 S_{3}(z)=\int_{-a}^{-b} g(t) \rho(t) d t \int_{2}^{\infty} e^{j K \xi} \lambda_{0}(\xi)\left[\frac{1}{t+j \xi}+\frac{1}{t-j \xi}\right] d \xi .
$$

To prove this we use the result that

$$
\int_{0}^{\infty} L(\eta, t) e^{-\xi \eta} d \eta=\lambda_{0}(\xi)\left[\frac{1}{t+j \xi}+\frac{1}{t-j \xi}\right]
$$

where

$$
L(\eta, t)=\frac{4 t}{\pi} \int_{-a}^{-b} \frac{\sin (s \eta)}{\rho(s)} \frac{d s}{t^{2}-s^{2}}
$$

and obtain that

$$
2 S_{3}(z)=\int_{-a}^{-b} g(t) \rho(t) d t \int_{z}^{\infty} e^{j K \xi} d \xi \int_{0}^{\infty} e^{-\xi \eta} L(\eta, t) d \eta
$$

which, after interchanging the orders of integration and simplification, gives the result that

$$
S_{3}(z)=\frac{e^{j K z}}{2} \int_{-a}^{-b} \rho(t) g(t) d t \int_{0}^{\infty} \frac{L(\eta, t)}{\eta-j K} e^{-\xi \eta} d \eta .
$$

Substituting the above results for $S_{1}(z), S_{2}(z)$ and $S_{3}(z)$ in (5.2) we ultimately find that the function $\phi_{1}(x, y)$ can be expressed as:

$$
\begin{aligned}
\phi_{1}(x, y)=T_{1} e^{K y+i K x}-B \int_{0}^{\infty} \frac{M(\eta)}{\eta^{2}+K^{2}} e^{-\eta x}[\eta \cos \eta y+K \sin \eta y] d \eta \\
+\frac{1}{\pi} \int_{-a}^{-b} f(t) d t \int_{0}^{\infty} \frac{\cos (\eta t)}{\eta^{2}+K^{2}} e^{-\eta x}[\eta \cos \eta y+K \sin \eta y] d \eta \\
+\frac{1}{2 \pi} \int_{-a}^{-b} g(t) \rho(t) d t \int_{0}^{\infty} \frac{L(\eta, t)}{\eta^{2}+K^{2}} e^{-\eta x}[\eta \cos \eta y+K \sin \eta y] d \eta \\
\text { for } x>0 .
\end{aligned}
$$

Note that if we use the result that $T_{1}=0$, the first term drops out.

The function $\phi_{1}(x, y)$ can be determined by a similar procedure for $x<0$ also, and we obtain that

$$
\phi_{1}(x, y)=R_{1} e^{K y-i K x}-R e_{j} e^{-j K z} \int_{z}^{\infty} e^{j K \xi} W_{1}(\xi) d \xi, \text { for } x<0,
$$


and this can be expressed ultimately as:

$$
\begin{gathered}
\phi_{1}(x, y)=R_{1} e^{K y-i K x}+B \int_{0}^{\infty} \frac{M(\eta)}{\eta^{2}+K^{2}} e^{\eta x}[\eta \cos \eta y+K \sin \eta y] d \eta \\
+\frac{1}{\pi} \int_{-a}^{-b} f(t) d t \int_{0}^{\infty} \frac{\cos (\eta t)}{\eta^{2}+K^{2}} e^{\eta x}[\eta \cos \eta y+K \sin \eta y] d \eta \\
-\frac{1}{2 \pi} \int_{-a}^{-b} g(t) \rho(t) d t \int_{0}^{\infty} \frac{L(\eta, t)}{\eta^{2}+K^{2}} e^{\eta x}[\eta \cos \eta y+K \sin \eta y] d \eta \\
\text { for } x<0 .
\end{gathered}
$$

The knowledge of the function $\phi_{1}(x, y)$ as given by (5.10) and (5.11) for $x>0$ and $x<0$ respectively, where $M(\eta)$ and $L(\eta, t)$ are as defined by (5.5) and (5.8), gives the idea of the complete field for this potential for all finite values of $x$.

It is observed that the above expressions (5.10) and (5.11) for the potential $\phi_{1}$ involve the functions $f$ and $g$ which, in tum, depend on the potential $\phi_{0}(0, y)$ $(y \in L)$, as well as the shape function $C(y)$. Therefore, these forms of $\phi_{1}$ are to be regarded as formal explicit expressions for the potential and their actual determination requires difficult but practicable manipulations which are not taken up in the present work.

\section{References}

[1] D.V. Evans, "Diffraction of water waves by a submerged vertical plate", J. Fluid Mech. 40 (1970) 433-451.

[2] B.N. Mandal and A. Chakrabarti, "A note on diffraction of water-waves by nearly vertical barriers", IMA. J. Appl. Math. 43 (1989) 157-165.

[3] B.N. Mandal and P.K. Kundu, "Scattering of waterwaves by a submerged nearly vertical plate", SIAM. J. Appl. Math. 50 (1990) 1221-1231.

[4] C.C. Mei, "Radiation and scattering of transient gravity waves by vertical plates", Quart. Journ. Mech. Appl. Math. 19 (1966) 415-440.

[5] N.I. Muskhelishvili, Singular integral equations, (Noordhoff, Groningen, 1953).

[6] D. Porter, "The transmission of surface waves through a gap in a vertical barrier", Proc. Camb. Phil. Soc. 71 (1972) 411-421.

[7] D.C. Shaw, "Perturbational results for diffraction of eater waves by nearly vertical barriers", IMA. J. Appl. Math. 34 (1985) 99-117.

[8] Stoker, Water waves, (Wiley Inter Science, 1957).

[9] F. Ursell, "The effect of a fixed vertical barrier on surface waves in deep water", Proc. Camb. Phil. Soc. 43 (1947) 374-382.

[10] L. Vijaya Bharathi and A. Chakrabarti, "Solution of a boundary value problem associated diffraction of water waves by a nearly vertical barrier", IMA. J. Appl. Math. 47 (1991) 23-32. 